\title{
Exercício com intensidade autosselecionada para idosos: implicações do afeto em aulas comunitárias
}

\author{
Self-selected intensity exercise for the elderly: implications of affect in \\ community classes
}

\begin{abstract}
AUTORES
Raul Cosme Ramos Prado ${ }^{1}$ (D)

Rodrigo Silveira ${ }^{1}$ (D)

Raul Canestri ${ }^{1}$ (iD

Hélio José Coelho-Junior ${ }^{1,2}$ (DD

Paulo Estêvão Franco-Alvarenga ${ }^{1}$ (D)

Cayque Brietzke ${ }^{1}$ (D)

Tony Meireles Santos ${ }^{1,3}$ (D)

Flávio Oliveira Pires ${ }^{1}$ (D)

Ricardo Yukio Asano ${ }^{1}$ (D)

1 Universidade de São Paulo, Escola de Artes Ciências e Humanidade, São Paulo, São Paulo, Brasil.

2 Universidade de Campinas, Laboratório de Cinesiologia Aplica, Campinas, São Paulo, Brasil.

3 Universidade Federal de Pernambuco, Departamento de Educação Física, Recife, Pernambuco, Brasil.

\section{CONTATO}

Raul Cosme Ramos Prado

raulprado@usp.br

Rua Arlindo Béttio, n. 1000, Vila Guaraciaba, São Paulo, São Paulo, Brasil.

CEP: 08810-040.

DOI
\end{abstract}

10.12820/rbafs. $24 \mathrm{e} 0089$

\section{(cc) BY-NC-SA}

Este obra está licenciado com uma Licença

Creative Commons Atribuição-NãoComercialCompartilhaIgual 4.0 Internacional.

\begin{abstract}
RESUMO
A prescrição da intensidade do exercício de forma autosselecionada em função de maior afeto positivo é uma estratégia utilizada para promover aderência da população na prática de exercícios. Entretanto, ainda não foram investigadas as implicações que essa estratégia pode gerar na valência afetiva de idosos em ambientes não laboratoriais e em aulas em grupos. $\mathrm{O}$ objetivo desse estudo foi analisar se o exercício com intensidade autosselecionada realizado em grupos de idosos pode influenciar na valência afetiva. A amostra foi composta por 176 idosos, sendo 42 homens, com média de idade $70,70 \pm 10,10$ anos e 134 mulheres, com média de idade 71,00 \pm 6,60 anos. Os idosos vinculados a um centro comunitário reportaram a valência afetiva e percepção subjetiva de esforço após uma caminhada de 30 minutos com intensidade autosselecionada. Os participantes foram alocados em três grupos segundo os tercis de percepção subjetiva de esforço: grupo com baixa (GBPE), média (GMPE) e alta (GAPE) percepção de esforço. Foi identificado que todos grupos se diferenciam entre si na valência afetiva $\left[\chi^{2}(2)=50,860 ; \mathrm{p}<0,05\right]$. A magnitude das diferenças pelo tamanho de efeito foi moderada na análise global ( $\left.{ }^{*} \mathrm{ES}=0,331\right)$ e entre GBPE e GAPE (ES =0,329). Conclui-se existe uma implicação negativa na valência afetiva em exercício com autosseleção da intensidade realizados por idosos em grupos comunitários, possivelmente causado por fatores psicossociais capazes de influenciar na variabilidade de respostas perceptivas.
\end{abstract}

Palavras-chave: Caminhada; Afeto; Saúde do idoso.

\section{ABSTRACT}

The self-selected exercise intensity prescription due to greater positive affect is a strategy used to promote adherence of the population to exercise. However, the implications that this strategy may have on the affective valence of the elderly in non-laboratory environments and in group classes have not still investigated. The purpose of this study was to analyze whether self-selected intensity exercise performed in elderly groups can influence affective valence. The sample consisted of 176 elderly, 42 men, with a mean age of $70.70 \pm$ 10.10 years and 134 women, with a mean age of $71.00 \pm 6.60$ years. The elderly linked to a community center reported affective valence and rating perceived exertion after a 30-minute walk with self-selected intensity. Participants were allocated to three groups according to the terciles of rating perceived exertion: group with low (GBPE), medium (GMPE) and high (GAPE) perceived exertion. It was identified that all groups differ in affective valence $\left[\chi^{2}(2)=50,860 ; p<0.05\right]$. The magnitude of the differences by effect size was moderate in the overall analysis ( $E S=0.331)$ and between $G B P E$ and $G A P E(E S=0.329)$. In conclusion, there is a negative implication in affective valence in self-selected exercise intensity performed by the elderly in community groups, possibly caused by psychosocial factors capable of influencing the variability of perceptual responses.

Keywords: Walking; Affect; Health of the elderly.

\section{Introdução}

O exercício com intensidade autosselecionada proporciona autonomia de escolha do próprio ritmo e considera as preferências individuais para sua prescrição ${ }^{1}$. Essa estratégia é comumente utilizada na prescrição de treinamento resistido ${ }^{2,3}$ e para ajustes na intensidade em exercícios aeróbios ${ }^{4}$.

Pesquisas demonstram que juntamente com o nível de aptidão física ${ }^{5}$ a genética ${ }^{6}$ e a preferência pelo tipo de exercício ${ }^{7}$, a autosseleção de intensidade também pode aumentar a valência afetiva (i.e., afeto) em comparação aos exercícios físicos realizados com intensidades impostas semelhantes ${ }^{8,9}$ ou quando a intensidade do exercício autosselecionado supera a intensidade do exercício com carga imposta9.

Essa estratégia atrelada a teoria hedonista considera 
que as pessoas poderão ter maior aderência em programas de exercício físico em função do maior afeto ${ }^{10}$. Outra vantagem dessa estratégia é que quando expostas ao modelo autosselecionado, os praticantes alcançam ou ultrapassam as recomendações mínimas de intensidade para benefícios a saúde ${ }^{1}$.

Essa estratégia foi investigada em diferentes subgrupos populacionais ${ }^{8,11,12}$. Em idosos foi observado em caminhada com intensidade autosselecionada de 20 minutos que os participantes ajustaram o ritmo ao longo da sessão, o que representou um aumento médio de $88,20 \pm 17,04 \%$ do consumo de oxigênio $\left(\mathrm{VO}_{2}-\mathrm{L} \cdot \mathrm{min}^{-1}\right)$ do limiar ventilatório nos primeiros 10 minutos ajustando para 107,70 $\pm 19,09 \%$ do $\mathrm{VO}_{2}\left(\mathrm{~L} \cdot \mathrm{min}^{-1}\right)$ do limiar ventilatório nos últimos 10 minutos, o que é reflexo do aumento da intensidade ao longo da caminhada ${ }^{12}$. Entretanto, a autosseleção da intensidade acima do limiar ventilatório favoreceu a manutenção das respostas afetivas positivas durante toda a sessão. Isso está associado ao instinto das pessoas em autosselecionar a intensidade do exercício em zonas de afeto positivo ${ }^{1}$.

O exercício com intensidade autosselecionada é uma estratégia capaz gerar um considerado impacto social. No entanto, a produção cientifica sobre o assunto se baseia em aplicação unicamente em ambientes laboratoriais ${ }^{12}$ e de forma individualizada onde o controle do ambiente é elevado, minimizando os equívocos dos participantes de subestimar ou superestimar as intensidades do exercício. Fato observado por Krinski et al. ${ }^{13}$ que identificaram menor percepção subjetiva de esforço (PSE) em um exercício realizado em ambiente externo quando comparado a mesma tarefa em ambiente controlado. Acredita-se que isso possa ter ocorrido por interações entre fatores ambientais que diminuem o foco no exercício e consequentemente geram a percepção de que o exercício no ambiente cotidiano seja mais fáci ${ }^{14}$.

Com isso, surge a necessidade de pesquisas que verifiquem a adequação da utilização do exercício com intensidade autosselecionada para prescrição de exercício físico em locais não laboratoriais e em grupos de idosos, uma vez que é crescente o número de programas de exercício físico em grupos comunitários direcionados a essa população ${ }^{15}$.

Em contra partida, isso também inclui os esclarecimentos de fatores psicossociais presente nesse contexto capazes de mudar o comportamento das pessoas, tal como o "efeito manada", que inconscientemente e impulsivamente faz com que as pessoas regulem seu ritmo de acordo com a cadência imposta por um grupo, in- cluindo na escolha da intensidade de uma caminhada ${ }^{16}$.

Desta forma, consta-se a necessidade de investigar as implicações da prescrição de exercício com intensidade autosselecionada na valência afetiva de idosos em ambientes não laboratoriais e em aulas em grupos. Uma vez que simplificar abordagens de prescrição de exercícios físicos frequentemente utilizadas aplicando essa estratégia poderá ter um elevado impacto social e validade externa. O presente estudo teve como objetivo analisar se o exercício com intensidade autosselecionada realizado em grupos de idosos pode influenciar na valência afetiva.

\section{Métodos}

Esta é uma pesquisa quase-experimental, sendo a seleção dos participantes realizada por conveniência. Todos os participantes assinaram o termo de consentimento livre e esclarecido (TCLE) que continha os objetivos, procedimentos, benefícios e riscos para integridade de cada um durante a pesquisa. $\mathrm{O}$ desenvolvimento da pesquisa seguiu a Declaração de Helsinki na resolução 466/12 do Conselho Nacional de Saúde juntamente com a autorização do Comitê de Ética e Pesquisa da Universidade de São Paulo sob o número de protocolo n 2.799 .925 e aprovação do ensaio pelo Registro Brasileiro de Ensaios Clínicos sob o número de registro RBR-4xxz7j.

Por meio de um convite, realizado durante as aulas com uma semana de antecedência das coletas, aproximadamente 1.000 idosos praticantes de exercício físico de um centro comunitário foram convidados para participar da pesquisa. Os participantes deveriam ter idade igual ou superior a 60 anos, no mínimo 6 meses de participação em exercícios físicos regulares no centro comunitário o que consequentemente vinha acompanhado da autorização médica para prática de exercícios físicos e não possuir comorbidades que interferissem em uma caminhada. Aos participantes foi informado que a participação era voluntária e que o teste iria ocorrer durante o período normal de aula. Além disso, que eles poderiam desistir a qualquer momento caso sentissem desconforto ou mal-estar. Por fim, foi destacado que a participação ou a desistência durante os protocolos da pesquisa não afetaria as aulas diárias ou qualquer outra futura oportunidade no centro comunitário.

No dia do teste os participantes tinham que estar no mínimo 24 horas sem realizar exercícios físicos ou consumo de bebidas alcoólicas, abster-se do uso de cafeína por no mínimo 3 horas antes da avaliação, ter realizado uma refeição leve previamente ao teste, deveriam estar com roupas confortáveis e com a frequência cardíaca de 
repouso (FCr), glicemia e pressão arterial controladas a partir da avaliação realizada antes da caminhada. Sem necessidade de exclusão de nenhum participante, 176 idosos aceitaram participar da pesquisa assinando TCLE.

A presente pesquisa foi conduzida no centro comunitário em que os participantes praticavam exercícios físicos. Em uma única visita com sessões médias de 45 minutos para cada grupo de 10 a 15 participantes.

Inicialmente no dia da coleta os participantes foram novamente familiarizados sobre os procedimentos da pesquisa e direcionados à uma área para a realização da avaliação antropométrica. Em seguida, os participantes foram direcionados para uma área em que permaneceram em repouso sentados em cadeiras por 5 minutos, durante esse período uma familiarização com a escala de valência afetiva e de PSE foi realizada para melhorar a interpretação e entendimento desses instrumentos para essa população. Durante os 30 segundos finais do período de repouso a $\mathrm{FCr}$, a pressão arterial e por fim a glicemia foram monitoradas como parâmetros para autorizar o início da caminhada.

Direcionados para um local com dimensões conhecidas $(20 \mathrm{~m} \times 10 \mathrm{~m})$ os participantes caminharam $30 \mathrm{mi}^{-}$ nutos com intensidade autosselecionada. A orientação para autosseleção da intensidade foi basicamente para que os participantes selecionassem a intensidade que eles desejassem e que conseguiriam cumprir durante os 30 minutos de caminhada, considerando que cada um poderia selecionar a sua própria intensidade de acordo com sua preferência. Além disso, os participantes foram orientados a não correr ou parar durante a caminhada e a realizar o teste sem competitividade. Para isso, um pesquisador foi responsável por monitorar a caminhada para que todos os procedimentos fossem cumpridos.

Imediatamente ao final dos 30 minutos, os idosos foram direcionados ao pesquisador responsável em apresentar as escalas e retratar uma pontuação média percebida na escala de valência afetiva e PSE frente a caminhada que tinham acabado de realizar. Individualmente os idosos retrataram sua percepção em cada escala, esse procedimento aconteceu pela ordem de chegada de cada participante até o pesquisador e teve a duração média de 25 segundos para cada grupo.

Para mensurar a massa corporal $(\mathrm{kg})$ foi utilizado a balança Solar Powered Bathroom Scales e para estatura $(\mathrm{cm})$ o estadiômetro da Filizola ${ }^{\circledR}$ (Brasil).

O monitoramento das medidas fisiológicas foi realizado para constatar que os idosos estavam em níveis fisiológicos adequados para realização da caminhada.
Inicialmente houve avaliação da frequência cardíaca pelo aparelho digital (Microlife.BP 3BT0-A ${ }^{\circledR}$, Finlândia) durante os últimos 30 segundos de um período de repouso de 5 minutos. Os idosos deveriam estar com a FCr entre 70 - $95 \mathrm{bpm}^{17}$, a pressão arterial sistólica (PAS) entre 110 - 159 mmHg; e a pressão arterial diastólica (PAD) entre $70-89 \mathrm{mmHg}^{17}$. Em seguida, a glicose sanguínea foi avaliada pelo glicosímetro Accu-Chek ${ }^{\circledR}$ Performa (Roche Diagnostics, BR), a glicemia sanguínea deveria estar entre $70-160 \mathrm{mg} / \mathrm{dL}^{18}$. O período de repouso foi suficiente para em uma única medida todos os participantes se enquadrarem no monitoramento das medidas fisiológicas.

Uma familiarização das escalas subjetivas foi cautelosamente realizada para não influenciar a resposta dos participantes, informando que não existia resposta certa ou errada no apontamento da escala.

A valência afetiva foi avaliada pela "Feeling Scale", sendo retratado o sentimento de prazer/afeto do participante frente ao momento. Essa escala não foi validada para a língua portuguesa, mas tem sido utilizada na comunidade científica nacional. A escala de valência afetiva consiste em uma estrutura bipolar de 11 pontos, sendo que em uma das extremidades, ancorada em resposta positiva de prazer/afeto era caracterizado um sentimento "muito bom" (+5) e na outra extremidade, a escala era ancorada na sensação de desprazer/desafeto com a caracterização de sentimento "muito ruim" $(-5)$. Entre as duas extremidades há o valor "0", caracterizando um sentimento "neutro".

Os participantes eram informados que o exercício tem a capacidade de proporcionar sensações prazerosas ou desprazerosas durante sua realização e a partir disso eles deveriam apontar na escala o ponto que mais sentiram durante a caminhada seguindo ancoragens como "quanto de sentimento você mais sentiu durante a caminhada?" ou "na escala de valência afetiva, qual ponto você mais sentiu durante a caminhada?".

A PSE foi avaliada por meio da escala proposta por Borg $^{20}$ que retrata a percepção de esforço do participante frente ao momento. A PSE consiste uma estrutura de 15 pontos que se inicia no número 6 que representa a percepção de "muito fácil", o que reflete em um baixo nível de esforço, e tem seu limite no ponto 20 que representa a percepção de "muito exaustivo", o que reflete em um alto nível de esforço ${ }^{21}$.

Os idosos foram solicitados a imaginar um estado de repouso representando um possível baixo nível de esforço em que uma caminhada, levantar uma sacola 
plástica pesada ou subir e descer degraus de escada possivelmente poderia aumentar o esforço percebido. Por fim, os participantes deveriam apontar na escala o ponto que mais sentiram durante a caminhada seguindo ancoragens como "quanto de esforço você mais sentiu durante a caminhada?" ou "quanto de esforço você sentiu com mais frequência para realizar a caminhada?”.

Os dados descritivos são apresentados em média, desvio padrão (DP), para todas as variáveis, exceto a valência afetiva que foi exibida em quartis, mediana e intervalo de confiança (IC 95\%), devido a não-normalidade dessa variável.

A normalidade e homogeneidade dos dados foram previamente verificadas, por meio do teste de Kolmogorov-Smirnov e Levene, respectivamente. Devido à distribuição não-normal dos dados foi utilizado a correlação de Spearman ( $r$ ) para avaliar a associação entre a PSE e a valência afetiva. Em seguida, os idosos foram estratificados em três grupos com base na PSE, sendo eles: grupo com baixa percepção subjetiva de esforço; PSE 6-11 (GBPE; n = 62) grupo com média percepção subjetiva de esforço; PSE 12 - 14 (GMPE; n = 72) e grupo com alta percepção subjetiva de esforço; PSE $15-20$ (GAPE; $n=42)$. Afim de verificar a homogeneidade dos grupos foi realizado um teste de Kruskal- Wallis para as variáveis fisiológicas (FCr, PAS, PAD e glicemia), o mesmo teste foi realizado para comparação da PSE e valência afetiva entre os grupos. Um teste de comparações múltiplas de Nemenyi foi realizado para localizar a diferença entre os grupos para as variáveis que sofreram efeito. Para medir a magnitude da diferença entre os grupos na valência afetiva foi utilizado o tamanho de efeito (ES) de Cohen's (d) adotando a clas- sificação de efeito pequeno ( $\mathrm{ES}=0,10)$, moderado (ES $=0,25)$ e grande $(\mathrm{ES}=0,40)^{22}$. A análise estatística dos dados foi realizada no software estatístico SPSS versão 23 , adotando-se um valor de $\mathrm{p}<0,05$.

\section{Resultados}

Todos os idosos que frequentavam o centro comunitário de exercício físico foram convidados a participar da pesquisa, contudo, sem a necessidade da exclusão de nenhum. Foram analisados dados de 176 idosos $(70,70$ $\pm 10,10$ anos), sendo 42 homens (75,00 $\pm 6,80$ anos) e 134 mulheres (71,00 $\pm 6,60$ anos) participaram da pesquisa. Cálculo de tamanho da amostra realizado a posteriori considerando uma estimativa de ES 0,40 e um $\alpha$ de 0,05 indicou que essa amostra tem poder de 0,95 .

$\mathrm{Na}$ Tabela 1 estão apresentadas as características da amostra e as respostas psicológicas após 30 minutos de caminhada com intensidade autosselecionada. Após a estratificação dos grupos, o GBPE foi composto por idosos com idade média de 71,22 \pm 7,67 anos; PSE média de 8,00 $\pm 1,37$; valência afetiva média de 3,32 $\pm 1,87$ com intervalo de respostas entre $\mathrm{L}_{\text {infe }}-4$ e $\mathrm{L}_{\text {supe }} 5$, já os idosos do GMPE tinham média de idade de 71,47 \pm 10,15 anos; PSE média de 12,53 $\pm 1,23$; valência afetiva média de 1,03 $\pm 3,30$ com intervalo de respostas entre $\mathrm{L}_{\text {infe }}-5$ e $\mathrm{L}_{\text {supe }} 5$, por fim, no GAPE os idosos tinham média de idade de 69,58 \pm 11,62 anos e PSE média de 16,62 $\pm 1,99$; valência afetiva média de $-1,3 \pm$ 2,45 e com intervalo de respostas entre $\mathrm{L}_{\text {infe }}-5$ e $\mathrm{L}_{\text {supe }} 5$.

Identificou-se que os grupos não apresentaram diferenças estatisticamente significativas para a $\mathrm{FCr}\left[\chi^{2}(2)=\right.$ $5,742 ; \mathrm{p}>0,05]$, PAS $\left[\chi^{2}(2)=1,293 ; p>0,05\right]$, PAD $\left[\chi^{2}\right.$ $(2)=0,656 ; p>0,05]$ e glicemia $\left[\chi^{2}(2)=4,437 ; p>0,05\right]$.

Tabela 1 - Variáveis antropométricas, fisiológicas e perceptivas de idosos, São Paulo, 2019 (n = 176).

\begin{tabular}{lcc}
\hline & GBPE & GBPE \\
\hline Variáveis & Média \pm DP & Média \pm DP \\
\hline Idade (anos) & $71,22 \pm 7,67$ & $71,47 \pm 10,15$ \\
Variáveis antropométricas e fisiológicas & & $69,58 \pm 11,62$ \\
Estatura (cm) & $1,66 \pm 0,04$ & $1,54 \pm 0,03$ \\
Massa corporal (kg) & $78,24 \pm 14,36$ & $67,45 \pm 12,04$ \\
Frequência cardíaca de repouso (bpm) & $70,46 \pm 16,37$ & $77,39 \pm 13,03$ \\
Glicemia (mg/dl) & $122,32 \pm 36,71$ & $119,88 \pm 32,62$ \\
PAS (mmHg) & $127,97 \pm 13,81$ & $125,72 \pm 16,64$ \\
PAD (mmHg) & $74,59 \pm 8,69$ & 76,02 \\
Variáveis perceptivas & & $76,06 \pm 9,41$ \\
Percepção subjetiva de esforço (u.a.) & $8,00 \pm 1,37$ & $122,75 \pm 9,5$ \\
Valência afetiva (u.a.) & $3,32 \pm 1,87$ & $12,53 \pm 1,23$ \\
\hline
\end{tabular}

Dados agrupados pelo GBPE = grupo com baixa percepção de esforço; GMPE = grupo com média percepção de esforço; GBPE = grupo com alta percepção de esforço; PAD = pressão arterial diastólica; PAS = pressão arterial sistólica; u.a. = unidade arbitrária. 
Foi constado diferença estatística significante entre todos os grupos para a PSE $\left[\chi^{2}(2)=155,135 ; \mathrm{p}<0,05\right]$.

Os resultados indicam que há correlação moderada e inversa $(r=-0,63 ; p<0,05)$ entre a PSE e a valência afetiva em idosos após uma caminhada com intensidade autosselecionada. Foi identificado um efeito significante dos grupos na valência afetiva $\left[\chi^{2}(2)=50,860 ; p\right.$ $<0,05]$. A partir do teste de comparações múltiplas de Nemenyi foi identificado que todos os grupos são diferentes na valência afetiva, com maiores valores para o $\operatorname{GBPE}(3,32 \pm 1,87)$; seguido por GMPE $(1,03 \pm 3,30)$ e GAPE $(-1,3 \pm 2,45)$. Foi encontrado um tamanho do efeito moderado para análise global ( $\left.{ }^{*} \mathrm{ES}=0,331\right)$ e para o GBPE vs GAPE (ES = 0,329), um tamanho do efeito pequeno para o GBPE vs GMPE $(\mathrm{ES}=0,179)$ e para o GMPE vs GAPE (ES = 0,179) - Figura 1.

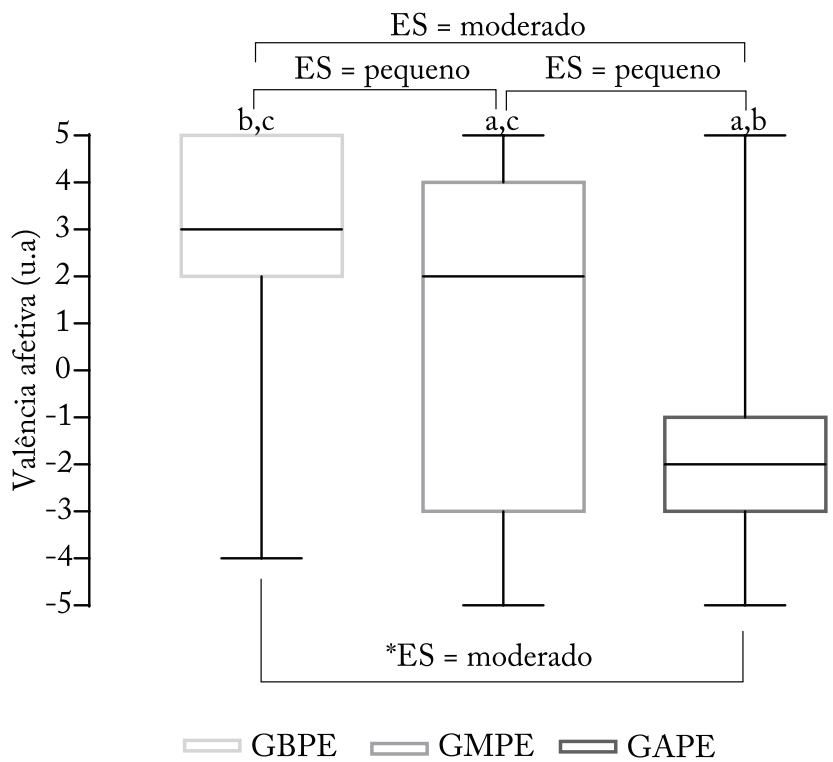

Figura 1 - Comparação da média valência afetiva entre os grupos estratificados pela PSE. Os dados são apresentados no boxplot em mediana e intervalo de confiança (IC 95\%), com análise de Kruskal-Wallis, comparação por pares de Nemenyi e ES da valência afetiva entre os grupos $(\mathrm{n}=176)$.

GBPE = grupo com baixa percepção de esforço; GMPE = grupo com média percepção de esforço; GBPE = grupo com alta percepção de esforço; a diferença significativa para o GBPE ( $p<0,05)$; b diferença significativa para o GMPE ( $\mathrm{p}<0,05)$; $\mathrm{c}$ diferença significativa para o GAPE $(p<0,05)$; ES = tamanho de efeito de Cohen's; *ES $=$ tamanho de efeito global.

\section{Discussão}

$\mathrm{Na}$ presente pesquisa foi possível identificar que os idosos foram heterogêneos na PSE e na valência afetiva, incluindo valores negativos de valência afetiva após a caminhada com intensidade autosselecionada.

Inicialmente os resultados de coeficiente de corre- lação entre a PSE e a valência afetiva corroboram com estudos prévios ${ }^{12,23}$, incluindo com idosos ${ }^{12}$ e a teoria do duplo modo ${ }^{24,25}$, em que a redução da valência afetiva é causada pelo aumento da PSE e de estresses fisiológicos provocado pelo aumento da intensidade de exercício.

Apesar de cada participante da presente pesquisa ter sido orientado a considerar sua preferência e desconsiderar qualquer interferência externa para autosseleção da intensidade da caminhada, foi identificada uma extensiva variabilidade de respostas perceptivas, incluindo grupo com mediana negativa de valência afetiva. Esses resultados são divergentes a um estudo prévio $^{12}$ que identificou que os idosos sempre autosselecionavam a intensidade em função da valência afetiva positiva.

A variabilidade dessas respostas percebidas podem ter ocorrido por características individuais de cada participante como a genética ${ }^{6}$, a preferência pelo modelo de exercício ${ }^{7}$ o nível aptidão física ${ }^{5}$ e/ou pelo "efeito manada", que leva uma pessoa a mudar seu comportamento de acordo com a direção que o grupo determina ${ }^{16}$, tal comportamento pode ter influenciado os idosos deste estudo a selecionarem a intensidade do exercício de acordo com o ritmo coletivo e não pela sua preferência, mesmo com a intensidade promovendo afeto negativo e elevada percepção de esforço.

Com isso, a extrapolação de resultados encontrados em ambientes laboratoriais ${ }^{12}$ para aulas comunitárias podem acarretar em distorções ao considerar as alterações em exercícios com autosseleção de intensidade em aulas em grupos.

Por sua vez, isso vai contra o conhecimento científico estabelecido, em que se acreditava que os idosos iriam autosselecionar a intensidade em função do afeto positivo $^{12}$, além disso, a elevada incidência de respostas negativas é preocupante por atrapalhar a aderência ao exercício físico nessa população.

Em contrapartida, variáveis que não foram controladas na presente pesquisa, incluindo preferências individuais pelo tipo de exercício, variáveis respiratórias, perfil do nível de aptidão física, podem ser consideradas variáveis de confundimento e terem influenciado cada idoso na autosseleção da intensidade do exercício. Por exemplo, Schineider \& Schmalbach ${ }^{26}$ identificaram que adolescentes autosselecionavam a intensidade do exercício, de acordo com o tamanho do benefício que a intensidade escolhida poderia impactar em sua saúde.

Desta forma, novas investigações envolvendo o exercício com intensidade autosselecionada para população idosa em ambientes comunitários devem ser de- 
senvolvidas suprindo as limitações deste estudo. Como a inclusão de um grupo controle com participantes que realizassem a mesma tarefa individualmente e em grupo, que não pratiquem exercícios aeróbios, com uma randomização para seleção dos participantes, controle das respostas perceptivas entre os participantes de cada grupo de teste para identificar se realmente existe influência do grupo para autosseleção da intensidade e o monitoramento de fatores que possam estar associados a autosseleção da intensidade da caminhada como a aptidão física e preferência pelo modelo de exercício.

Por outro lado, a presente pesquisa mostra-se com grande impacto social na diminuição da inatividade física devido a progressão do conhecimento científico voltado ao exercício com intensidade autosselecionada para população idosa em ambientes comunitários. Isso inclui que esse modelo de prescrição de exercício pode sofrer implicações em sua aplicação entre o ambiente do dia-a-dia e o laboratório.

Conclui-se que há uma implicação negativa da valência afetiva em exercícios realizados com intensidade autosselecionada por idosos em grupos comunitários, identificado pela variabilidade de respostas perceptivas durante a caminhada com intensidade autosselecionada devido a uma possível influência do grupo.

\section{Conflito de interesses}

Os autores declaram que não há conflito de interesses.

\section{Contribuição dos autores}

Prado RCR, Silveira R, Coelho-Junior HJ participaram na coleta de dados. Prado RCR, Silveira R, Canestri R, Coelho-Junior HJ, Franco-Alvarenga PE e Brietzke C, Santos TM, Pires FO e Asano RY participaram na concepção inicial do estudo e na revisão.

\section{Agradecimentos}

Ao apoio financeiro da Coordenação de Aperfeiçoamento de Pessoal de Nível Superior (CAPES-Brasil), do Código Financeiro 001 (Raul Cosme Ramos Prado. Rodrigo Silveira, Raul Canestri, Hélio José Coelho-Júnior, Paulo Estevão Franco-Alvarenga e Cayque Brietzke).

\section{Referências}

1. Ekkekakis P. Let them roam free? Physiological and psychological evidence for the potential of selfselected exercise intensity in public health. Sports Med. 2009;39(10):857-88.

2. Elsangedy HM, Machado DGS, Krinski K, Nascimento PHD, Oliveira GTA, Santos TM, et al. Let the Pleasure Guide Your Resistance Training Intensity. Med Sci Sports Exerc. 2018;50(7):1472-9.
3. Portugal EMM, Lattari E, Santos TM, Deslandes AC. Affective Responses To Prescribed And Self-Selected Strength Training Intensities. Percept Mot Skills. 2015;121(2):465-81.

4. Oliveira BRR, Deslandes AC, Thompson WR, Terra BS, Santos TM. Comparison of two proposed guidelines for aerobic training sessions. Percept Mot Skills. 2012;115(2):645-60.

5. Magnan RE, Kwan BM,Bryan AD. Effects of current physical activity on affective response to exercise: physical and socialcognitive mechanisms. Psychol Health. 2013;28(4):418-33.

6. Karoly H, Stevens C, Magnan R, Harlaar N, Hutchison $\mathrm{KE}$, Bryan AD. Genetic Influences on Physiological and Subjective Responses to an Aerobic Exercise Session among Sedentary Adults. J Cancer Epidemiol. 2012;2012:540563.

7. Kriel Y, Askew CD, Solomon C. The effect of running versus cycling high-intensity intermittent exercise on local tissue oxygenation and perceived enjoyment in 18-30-year-old sedentary men. PeerJ. 2018;19(6):e5026.

8. Hamlyn-Williams CC, Freeman P, Parfitt G. Acute affective responses to prescribed and self-selected exercise sessions in adolescent girls: an observational study. BMC Sports Sci Med Rehabil. 2014;25(6):35.

9. Oliveira BRR, Santos TM, KilpatrickM, Pires FO, Deslandes AC. Affective and enjoyment responses in high intensity interval training and continuous training: A systematic review and meta-analysis. PLoS One. 2018;13(6):0197124.

10. Brickman P, Campbell DT. Hedonic relativism and planning the good society. In: New York: Academic Press. 1971;287301.

11. Kellogg E, Cantacessi C, McNamer O, Holmes H, von Bargen R, Ramirez R, et al. Comparison of Psychological and Physiological Responses to Imposed vs. Self-selected High-Intensity Interval Training. J strength Cond Res. 2018; 33(11):2945-52.

12. Smith AE, Eston R, Tempest GD, Norton B, Parfitt G. Patterning of physiological and affective responses in older active adults during a maximal graded exercise test and selfselected exercise. Eur J Appl Physiol. 2015;115(9):1855-66.

13. Krinski K, Machado DS, Lirani L, DaSilva SG, Costa E, Hardcastle S, et al. Let's Walk Outdoors! Self-Paced Walking Outdoors Improves Future Intention to Exercise in Women With Obesity.J Sport and Exerc Psychol. 2017;39(2):145-57.

14. Gladwell VF, Brown DK, Wood C, Sandercock GR, Barton JL. The great outdoors: how a green exercise environment can benefit all. Extrem Physiol Med. 2013;2(1):3-3.

15. Tuso P. Strategies to Increase Physical Activity. Perm J. 2015;19(4):84-8.

16. Raafat RM, Chater N, Frith C. Herding in humans. Trends Cogn Sci. 2009;13(10):420-28.

17. Carey RM, Whelton PK. Prevention, Detection, Evaluation, and Management of High Blood Pressure in Adults: Synopsis of the 2017 American College of Cardiology/ American Heart Association Hypertension Guideline. Ann Intern Med. 2018;168(5):351-58.

18. Diabetes AA. lassification and Diagnosis of Diabetes: Standards of Medical Care in Diabetes. Diabetes Care. 2018;1;41(Suppl 1):S13-27.

19. Hardy C, Rejeski W. Not what, but how one feels: the measurement of affect during exercise. J Sport Exerc Psychol. 1989;1;11(3):304-17.

20. Borg GAV. Psychophysical bases of perceived exertion. Med Sci Sport Exerc. 1982;14(5):377-81. 
21. Pires FO, Noakes TD, Lima-Silva AE, Bertuzzi R, Ugrinowitsch C, Lira FS, et al. Cardiopulmonary, blood metabolite and rating of perceived exertion responses to constant exercises performed at different intensities until exhaustion. Br J Sports Med. 2011;45(14):1119-25.

22. Cohen J. A power primer. Psychol Bull. 1992;112(1):155-59.

23. Vasconcelos G, Canestri R, Prado RCR, Brietzke C, FrancoAlvarenga P, Santos TM, et al. A comprehensive integrative perspective of the anaerobic threshold engine. Physiol Behav. 2019;15(210):112435-38.

24. Ekkekakis P. Pleasure and displeasure from the body: Perspectives from exercise. Cogn Emot. 2003;17(2):213-39.
25. Ekkekakis P, Acevedo E. Affective responses to acute exercise: toward a psychobiological dose-response model. In: In E. O. Acevedo \& P. Ekkekakis (Eds.), Psychobiology of Physical Activity. United States of America. Human Kine.. 2006;91-109.

26. Schneider M, Schmalbach P. Affective Response to Exercise and Preferred Exercise Intensity Among Adolescents. J Phys Act Health. 2015;12(4):546-52.

Recebido: $21 / 02 / 2019$

Aprovado: 20/11/2019

\section{Como citar este artigo:}

Prado RCR, Silveira R, Canestri R, Coelho-Junior HJ, Franco-Alvarenga PE, Brietzke C, Santos TM, Pires FO, Asano RY. Exercício com intensidade autosselecionada para idosos: implicaçōes do afeto em aulas comunitárias. Rev Bras Ativ Fis Saúde. 2019;24:e0089. DOI: $10.12820 /$ rbafs. 24 e0089 